\title{
Commentary: Bleeding events with novel bioprostheses: Still a mystery
}

\author{
Manuel J. Antunes, MD, PhD, DSc
}

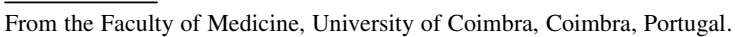

Disclosures: Author has nothing to disclose with regard to commercial support.

Received for publication Oct 8, 2019; revisions received Oct 8, 2019; accepted for publication Oct 8, 2019; available ahead of print Oct 24, 2019.

Address for reprints: Manuel J. Antunes, MD, PhD, DSc, Faculty of Medicine of the University of Coimbra, 3000075 Coimbra Portugal (E-mail: mjantunes48@sapo.pt).

J Thorac Cardiovasc Surg 2021;161:78-9

0022-5223/\$36.00

Copyright (C 2019 by The American Association for Thoracic Surgery

https://doi.org/10.1016/j.jtcvs.2019.10.052

In 2018, Sabik and colleagues ${ }^{1}$ published in this Journal the 1-year results of the Pericardial Surgical Aortic Valve Replacement (PERIGON) Pivotal Trial for the Medtronic Avalus valve (Medtronic, Inc, Minneapolis, Minn) in 864 patients who underwent aortic valve replacement with this novel prosthesis. In this report, the authors showed that the Medtronic Avalus valve had rates of adverse events that they considered comparable with those of other contemporary pericardial aortic valves (all-cause death was $7.2 \%$, valve-related in $1.1 \%$ ), but greater-thanexpected bleeding rates. The authors then hypothesized that the greater bleeding rate was related to patient medication and to associated conditions.

The objective of the current manuscript, from the same group of authors, was to explore bleeding events within 3 years postimplantation in 1115 patients meanwhile included in the trial. ${ }^{2}$ The authors analyzed frequency, timing, and severity of bleeding events, focusing on patients taking antiplatelet/anticoagulant medications at the time of the event. The conclusions are for an all-hemorrhage rate of $8.7 \%$ and major-hemorrhage of $5.2 \%$, with most events occurring late ( $>30$ days postsurgery). The linearized rate of bleeding (1.83\% patient/year) was more than twice that of the objective performance criteria (OPC) defined for this complication. The percentage of patients taking an antithrombotic drug between surgery and 30 days, between 30 days and 1 year, and after 1 year was $51.3 \%$, $20 \%$ and $19.6 \%$, respectively. The number of patients who had a major bleed at the same time intervals was $11 \%, 26 \%$, and $16 \%$. Only $10 \%$ of the 51 major bleeding events occurred in patients who were taking anticoagulant/ antiplatelet medication for post-aortic valve replacement prophylaxis; the remaining were on antithrombotic medication for other indications. Age, peripheral vascular disease, renal dysfunction, and dual antiplatelet use at baseline were predictors of all late bleeds.

Apparently, there does not seem to be a strong correlation between the proportion of patients taking an antithrombotic

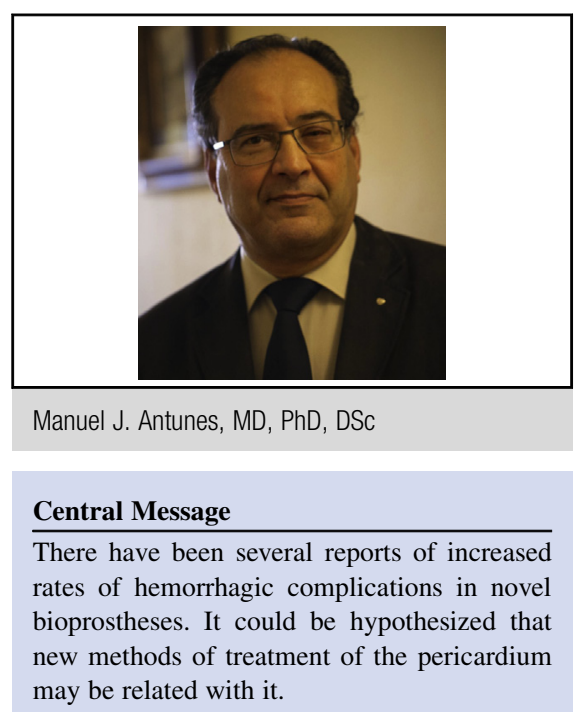

See Article page 66.

drug and the absolute numbers of patients presenting major bleeding. In addition, the percentage of patients with late major bleeding events (>30 day) taking aspirin or another antiplatelet agent was roughly one half of those with no late major bleeding who were taking single antiplatelet therapy $(25.6 \%$ vs $48.5 \%)$, and the rate of patients on anticoagulation therapy was the same (around 9\%). Most bleeding events occurred in patients taking antiplatelet and/or anticoagulation for indications other than postimplant prophylaxis, which are unavoidable. Interestingly, only double antiplatelet therapy came out as significant in the Cox regression analysis, but not the use of anticoagulants. Hence, the mechanism behind the observed high bleeding rate is more complex, probably a combination of baseline patient characteristics/fragilities and treatment effect. However, it could also be related to the prosthesis itself.

Seven patients died within 30 days of a major bleeding event (range, 0-15 days), which renders bleeding a very serious complication. The authors considered that there was a "low mortality" in this series. Their perioperative mortality was $1.2 \%$, but a global mortality of $7.3 \%$ at 3 years (the 3-year follow-up was not completed for all participating patients) may not be that low and may be related to the complication under study. This requires further analysis. In this regard, it is relevant to stress that this was a relatively young and low-risk population (mean 
age 70.2 years; Society of Thoracic Surgeons score 2.0), even considering that concomitant procedures were performed in approximately one half of the patients.

Other bioprostheses have recently been associated with this problem, and its genesis remains obscure. ${ }^{3}$ It is clear that the authors consider the incidence of hemorrhage, especially major episodes, in excess of the OPC but, apparently, they do not associate it with the prosthesis. They state that "several recent-generation surgical tissue valves have been found to have bleeding rates exceeding those recommended by regulatory bodies." However, it is not clear whether the values for the Avalus are greater than those observed with other modern pericardial prostheses. The authors tried very hard to justify the excessive bleeding events with this prosthesis, claiming for possible subjective differences in the classification of the events but, again, the comparison with other valves is not clear. They claim for a revision of the OPC, which they consider outdated. My view is that they should call for a revision of their antiplatelet/anticoagulation protocols. Indeed, during the process of revision of the manuscript, the authors conceded that "this may suggest that the use of antithrombotic therapy was too aggressive and current protocols require revision."

The authors also analyzed the hemodynamic evolution of the valves and other outcomes that, judging by the title, appears out of the scope of the paper. However, significant patient-prosthesis mismatch in $30 \%$ of patients was initially identified, which may raise questions about the adequacy of the surgical technique and experience of the surgeons performing the procedure, and a few cases of increasing gradients were found in the follow-up. Could they, in any way, be related to incipient valve leaflet thrombosis and the use/non-use of anti-aggregation? The rate of structural valve disease in the PERIGON trial is very low, probably due to the as yet-short follow-up, to reliably analyze the effect of anticoagulation therapy on its incidence.

In conclusion, there have been several reports of increased rates of hemorrhagic complications in recently marketed bioprostheses, some related to low platelet counts, although not always associated with any major postoperative complication. ${ }^{4}$ This phenomenon had not been previously described with previous generation bioprostheses. One could hypothesize that new methods of treatment of the pericardium may have something to do with it, but the use of anti-aggregation may remain the most important in its genesis. In any case, there cannot be smoke without fire. This matter needs further investigation.

\section{References}

1. Sabik JF III, Rao V, Lange R, Kappetein AP, Dagenais F, Labrousse L, et al. Oneyear outcomes associated with a novel stented bovine pericardial aortic bioprosthesis. J Thorac Cardiovasc Surg. 2018;156:1368-77.

2. Klautz RJM, Vriesendorp MD, Dagenais F, Labrousse L, Bapat V, Moront M, et al Antithrombotic therapy and bleeding events after aortic valve replacement with a novel bioprosthesis. J Thorac Cardiovasc Surg. 2021;161:66-75.e4.

3. Kizilay M, Elbir F, Aglar AA, Vural U, Balci AY, Yekeler İ. An overlooked fact: thrombocytopenia following bioprosthetic aortic valve replacement. Kardiochir Torakochirurgia Pol. 2019;16:19-26.

4. Stanger O, Grabherr M, Gahl B, Longnus S, Meinitzer A, Fiedler M, et al. Thrombocytopaenia after aortic valve replacement with stented, stentless and sutureless bioprostheses. Eur J Cardiothorac Surg. 2017;51:340-6. 